\title{
Métodos de avaliação da composição corporal em crianças
}

\author{
Methods of body composition evaluation in children
}

Mônica de Souza L. Sant'Anna', Silvia Eloíza Priore², Sylvia do Carmo C. Franceschini²

RESUMO

Objetivo: Revisar as principais técnicas de avaliação da composição corporal de crianças, enfatizando os estudos de validação.

Fontes de dados: Foi realizada uma busca nas principais bases científicas em saúde (SciELO, Science Direct e Pubmed), utilizando-se os descritores: "validação”, “composição corporal”, "criança”, "índice de massa corporal”, "pesagem hidrostática”, "plestimografia”, "bioimpedância elétrica”, "pregas cutâneas", "antropometria”, "circunferência da cintura" e respectivos vocábulos em inglês e espanhol. Foram selecionados artigos publicados entre 1990 e 2007 e trabalhos clássicos referentes ao tema, publicados anteriormente.

Síntese dos dados: Os métodos indiretos mais utilizados para avaliação da composição corporal de crianças são a plestimografia e a absortometria radiológica de dupla energia. Dentre os métodos duplamente indiretos, os mais utilizados são o índice de massa corporal, as pregas cutâneas, a bioimpedância elétrica, a circunferência da cintura, a relação cintura/ quadril, o índice de conicidade e a relação cintura/estatura, sendo que as maiores correlações encontradas foram para a bioimpedância elétrica e o índice de massa corporal.

Conclusões: Existem vários métodos para avaliar a composição corporal em crianças, cada um com suas vantagens e desvantagens. Ao definir o melhor método, é preciso eleger aquele que melhor detecte o problema que se pretende corrigir, levando em consideração os custos, o nível de treinamento dos avaliadores, o tempo de execução, a receptividade da população e os possíveis riscos à saúde.

Palavras-chave: composição corporal; criança; antropometria; avaliação nutricional.

\section{ABSTRACT}

Objective: To review the main methods to assess body composition of children, emphasizing validation studies.

Data sources: SciELO, Science Direct and Pubmed databases were searched using the following key-words: "validation", "body composition", "child”, "body mass index", "hydrostatic weighing”, "plesthymography", "electrical bioimpedance", "skinfold, anthropometry, waist circumference. Studies published between 1990 and 2007 in English and Spanish, as well as recognized classical works on the theme, were selected.

Data synthesis: The most widely used indirect methods to evaluate the body composition of children were plesthymography and dual energy X-ray absorptiometry. Among the double indirect methods, the most frequently used ones were body mass index, skinfold thickness, electrical bioimpedance, waist circumference, waist-to-hip ratio, conicity index and waist-to-height ratio. Higher correlations were found for electrical bioimpedance and body mass index.

Conclusions: There are many methods to evaluate a child's body composition, each one with advantages and disadvantages. Before starting the evaluation, an effective method must be chosen to identify the problem, considering costs, the evaluators' skills, timing, acceptance by the target population and possible risks to health.

Key-words: body composition; child; anthropometry; nutrition assessment.
Instituição: Universidade Federal de Viçosa (UFV), Viçosa, MG, Brasil ${ }^{1}$ Mestre em Ciência da Nutrição pelo Departamento de Nutrição e Saúde da UFV, Viçosa, MG, Brasil

Doutora em Nutrição; Docente do Departamento de Nutrição e Saúde da UFV, Viçosa, MG, Brasil
Endereço para correspondência:

Mônica de Souza L. Sant'Anna

Avenida Santa Rita, 216/101 - Centro

CEP 36570-000 - Viçosa/MG

E-mail: mslsantana@yahoo.com

Recebido em: 3/8/08

Aprovado em: 6/11/08 


\section{Introdução}

Medidas de composição corporal podem ser utilizadas para avaliar tanto padrões de crescimento e desenvolvimento quanto para quantificar a gordura corporal relativa em crianças $^{(1)}$. O aumento da prevalência da obesidade infantil e o fato de se tratar de um fator de risco para a obesidade adulta, além de preditor de doenças cardiovasculares, torna a avaliação cuidadosa da composição corporal na criança uma importante variável de promoção da saúde ${ }^{(2)}$.

O interesse em medir a quantidade dos diferentes componentes do corpo humano iniciou-se no século 19 e aumentou no final do século 20 devido à associação entre o excesso de gordura corporal e o aumento do risco de desenvolvimento de doenças coronarianas, diabetes melito tipo 2, ósteo-artrites e até mesmo alguns tipos de câncer ${ }^{(3)}$.

A té o início do século 20, a análise da composição corporal ainda era feita por meio de dissecação de cadáveres, considerada até hoje a única maneira direta de medir os principais componentes do corpo humano. Em 1940, Behnke iniciou estudos que objetivavam estabelecer métodos indiretos para determinar a composição corporal. Os trabalhos pioneiros de Behnke e Brozek obtiveram dois resultados ainda válidos: o estabelecimento da pesagem hidrostática como padrão-ouro para todos os outros métodos indiretos e o estabelecimento do modelo de dois componentes (peso gordo e peso magro) como base para estudos de composição corporal ${ }^{(4)}$.

A maioria dos métodos utilizados para avaliar a composição corporal é validada em adultos, sendo em menor número os estudos de validação em crianças. Este artigo tem como objetivo revisar as técnicas mais utilizadas de análise de composição corporal em crianças, enfocando os estudos de validação desses métodos para o referido grupo etário.

\section{Métodos}

Foi realizada uma busca nas principais bases científicas em saúde (SciELO, Science Direct e Pubmed) com a utilização dos seguintes descritores: "validação", "composição corporal", "criança", "índice de massa corporal”, "pesagem hidrostática”, "plestimografia”, "bioimpedância elétrica”, "pregas cutâneas", "antropometria", "circunferência da cintura", com respectivos vocábulos em inglês e espanhol. As expressões de pesquisa foram construídas combinando-se esses termos ou utilizando-os de forma isolada. Artigos referenciados em outros estudos também foram consultados. Selecionaram-se artigos publicados entre 1990 e 2007 e trabalhos clássicos referentes ao tema, publicados anteriormente. Dos 265 artigos identificados, foram selecionadas 48 publicações de acordo com os objetivos da revisão.

\section{Técnicas de análise da composição corporal}

Segundo Martin e Drinkwater ${ }^{(5)}$, essas técnicas são divididas em três grupos: diretas, indiretas e duplamente indiretas. O método direto, apesar de apresentar elevada precisão, tem utilidade limitada, pois a análise é realizada por dissecação física ou físico-química de cadáveres.

\section{Análise indireta da composição corporal}

As técnicas indiretas são precisas, possuem uma limitada aplicação prática e um alto custo financeiro. São utilizadas principalmente para validar as técnicas duplamente indiretas. No presente artigo, serão revisadas as seguintes técnicas de análise indireta da composição corporal: pesagem hidrostática, hidrometria, plestimografia e absortometria radiológica de dupla energia (DEXA). Existem outras técnicas indiretas de avaliação da composição corporal, porém só foram contempladas as supracitadas por serem as mais utilizadas em crianças.

\section{Pesagem hidrostática}

A pesagem hidrostática é considerada o padrão-ouro na análise da composição corporal. Sua fidedignidade com a dissecação de cadáveres é excelente $(r=0,99)^{(6)}$. Esta técnica considera que o corpo é formado por dois componentes distintos: massa de gordura e massa livre de $\operatorname{gordura}^{(7)}$. A massa de gordura é constituída por todos os lipídeos que são extraíveis e a massa livre de gordura inclui água, proteína e componentes minerais ${ }^{(8)}$. A densidade corporal é determinada por meio da relação do peso no ar e o peso na água ${ }^{(9)}$. Sabendo-se o valor da densidade corporal, é possível estimar o percentual de gordura corporal por meio dos modelos matemáticos de $\operatorname{Siri}^{(10)} \mathrm{e} \mathrm{Brozek}^{(11)}$. Tais modelos foram baseados nos estudos iniciais de cadáveres, os quais supõem que a massa livre de gordura é constante. Nas crianças, este modelo é especialmente limitado devido às alterações nas proporções e densidade dos componentes da massa livre de gordura, que ocorrem durante o crescimento e maturação, evidenciadas principalmente pela redução na água corporal total e pelo aumento do conteúdo mineral $^{(8)}$. Além disso, esta técnica requer uma grande cooperação do avaliado, tornando-a inviável para lactentes e pré-escolares. 


\section{Hidrometria}

A hidrometria é um método invasivo de estimativa da água corporal total, devido à necessidade de ingerir ou aplicar uma substância (isótopo de hidrogênio) no indivíduo, a qual será distribuída igualmente por toda a água contida no $\operatorname{corpo}^{(12)}$. O método mais comum de medir a água corporal total em crianças é o da diluição de isótopos, podendo ser utilizados isótopos estáveis de deutério $\left(\mathrm{D}_{2} \mathrm{O}\right)$ ou oxigênio $18\left(\mathrm{H}_{2}{ }^{18} \mathrm{O}\right)$. $\mathrm{O}$ deutério superestima a água corporal total em torno de 0,5 a $5 \%$, contudo é mais utilizado por possuir custo mais baixo do que o oxigênio 18. É preciso empregar constantes de hidratação de acordo com sexo e com a idade ${ }^{(8)}$.

\section{Plestimografia}

A plestimografia estima o volume corporal por meio do deslocamento de $\mathrm{ar}^{(13)}$. Este método utiliza a relação inversa entre pressão e volume com base na lei de Boyle para determinar o volume corporal. Definido esse volume, é possível aplicar os princípios da densitometria para determinar a composição corporal por meio do cálculo da densidade corporal $^{(14)}$. O aparelho de plestimografia é feito de fibra de vidro e acoplado a um computador, o qual determina as variações no volume de ar e de pressão no interior para a câmara vazia e ocupada, fazendo ajustes para variáveis pulmonares necessárias na estimativa do volume corporal ${ }^{(15)}$.

Durante a avaliação, é importante que o indivíduo esteja descalço e usando o mínimo de roupa possível para não haver disparidades ${ }^{(14,16)}$. Vários estudos têm demonstrado a validade dos resultados de composição corporal apresentados pela plestimografia em comparação à pesagem hidrostática, determinando assim a validade deste método para diferentes populações ${ }^{(14,17,18)}$.

A plestimografia é um método de determinação da composição corporal rápido e fácil, depende menos da cooperação do avaliado e possui menor duração (três a cinco minutos contra 30 a 60 minutos da pesagem hidrostática) ${ }^{(12)}$.

Demerath et $a l^{(19)}$ avaliaram a validade e a reprodutibilidade da plestimografia em relação à pesagem hidrostática. Foram avaliadas 39 crianças e adolescentes com idade entre oito e 17 anos. Para avaliar a reprodutibilidade do método, os participantes foram avaliados dois dias consecutivos. O coeficiente de reprodutibilidade (CR) foi $90 \%$, sendo maior nas meninas ( $\mathrm{CR}=93 \%)$ do que nos meninos ( $\mathrm{CR}=87 \%)$, com coeficiente de variação de 6,5 e 10,8\%, respectivamente. A correlação encontrada entre os dois métodos para a densidade corporal foi 0,75 e, para o porcentual de gordura corporal, foi 0,76 , com erro padrão de estimativa para esse último porcentual de $4,3 \%$.
A plestimografia apresentou uma tendência a superestimar o porcentual de gordura corporal nos indivíduos com maior proporção de gordura e a subestimar tal porcentual naqueles com menor proporção de gordura no corpo.

Em outro estudo, Ma et al ${ }^{(20)}$ avaliaram 36 crianças pela plestimografia e pela hidrometria. Os autores não encontraram diferença significativa na mensuração do porcentual de gordura corporal entre os dois métodos $\left(\mathrm{R}^{2}=0,76\right)$. $\mathrm{O}$ erro padrão estimado encontrado neste estudo foi de $3,3 \%$.

Nos dois estudos, os autores concluem que a plestimografia pode ser utilizada para avaliar a composição corporal em crianças em substituição à pesagem hidrostática e à hidrometria por ser menos invasiva e depender em menor escala da cooperação do avaliado.

\section{Absortometria radiológica de dupla energia (DEXA)}

O DEXA é uma técnica de "escaneamento" que mede diferentes atenuações de dois raios X que passam pelo corpo ${ }^{(21)}$. Os raios X são emitidos por uma fonte que passa por baixo do indivíduo, o qual permanece em posição supina sobre a mesa. Após passar pelo indivíduo, os raios $\mathrm{X}$ atenuados são medidos por um detector discriminante de energia. O DEXA faz análises transversas do corpo, em intervalos de $1 \mathrm{~cm}$ da cabeça aos pés. Esta é uma técnica não invasiva considerada segura e que pode medir três componentes corporais: massa de gordura, massa livre de gordura e massa óssea ${ }^{(8)}$.

Segundo Bottaro et al ${ }^{(22)}$, o DEXA é uma tecnologia que vem sendo recentemente reconhecida como método de referência na análise da composição corporal. Alguns fatores limitantes, como custo elevado do equipamento e exposição à radiação, devem ser levados em consideração em se tratando de crianças.

Sopher $e t a l^{(23)}$ compararam o porcentual de gordura corporal medido pelo DEXA e pelo modelo de quatro compartimentos (4C) em 411 crianças e adolescentes com idade entre seis e 18 anos. Os autores encontraram uma diferença no porcentual de gordura corporal medido pelos dois métodos, mas com uma forte correlação $\left(\mathrm{R}^{2}=0,85\right)$ e erro padrão estimado em 3,6\%. Neste estudo, o DEXA tendeu a superestimar o porcentual de gordura corporal nos indivíduos com alta porcentagem de gordura e a subestimá-la naqueles com baixa porcentagem de gordura. A etnia, o gênero, a idade, o estágio de maturação sexual, o peso, a altura e o índice de massa corporal (IMC) não afetaram a relação entre os dois métodos. Os autores concluíram que o DEXA deve ser utilizado em estudos epidemiológicos e clínicos para avaliar o porcentual de gordura corporal em crianças com o intuito de atenuar as desordens metabólicas causadas pelo excesso de gordura. 


\section{Análise duplamente indireta da composição corporal}

Procedimentos laboratoriais oferecem estimativas muito precisas sobre os componentes de massa de gordura e massa livre de gordura sendo a primeira opção para a análise de composição corporal. No entanto, em razão do alto custo dos equipamentos, da sofisticação metodológica e das dificuldades em envolver os avaliados nos protocolos de medida, sua utilização tem sido limitada. Assim, houve maior encorajamento ao emprego do método antropométrico para analisar a composição corporal devido à sua simplicidade, inocuidade, relativa facilidade de interpretação e menores restrições culturais ${ }^{(24)}$.

As técnicas duplamente indiretas são menos rigorosas, porém apresentam melhor aplicação prática e menor custo financeiro, podendo ser empregadas tanto em pesquisas de campo quantos em estudos clínicos. Neste grupo, destacamse a bioimpedância elétrica e a antropometria, incluindo o IMC, as pregas cutâneas, as medidas de perímetros (circunferência da cintura, relação cintura/quadril), o índice de conicidade e a relação cintura/estatura.

\section{Bioimpedância elétrica (BIA)}

Este método se baseia na condução de uma corrente elétrica de baixa intensidade através do corpo. A impedância ou resistência ao fluxo da corrente elétrica é medida pela bioimpedância elétrica. Como a impedância varia de acordo com o tecido que está sendo mensurado e, sendo a massa magra um bom condutor de energia por possuir alta concentração de água e eletrólitos e a massa gorda um mau condutor de energia, pode-se dizer que a impedância é diretamente proporcional ao porcentual de gordura corporal ${ }^{(6)}$.

A validade e a precisão do método de bioimpedância elétrica são influenciadas por vários fatores como tipo de instrumento, colocação do eletrodo, nível de hidratação, alimentação, ciclo menstrual, temperatura ambiente e equação de predição ${ }^{(3)}$. Várias equações foram desenvolvidas e validadas com um $\mathrm{r}$ variando de 0,60 a 0,98 e um erro padrão estimado de 1,37 a $3,47^{(25)}$. Assim, para não comprometer o resultado da análise da composição corporal pela bioimpedância elétrica, alguns cuidados prévios são importantes: não comer ou beber quatro horas antes do teste, não fazer exercícios 12 horas antes do teste, urinar 30 minutos antes do teste, não consumir álcool nas 24 horas anteriores ao teste e não ter feito uso de medicamentos diuréticos nos últimos sete $\operatorname{dias}^{(3)}$.
Wu et a ${ }^{(26)}$ avaliaram 47 crianças e adolescentes com idade entre oito e 20 anos pela bioimpedância elétrica e pela pesagem hidrostática e encontraram uma correlação de 0,98 nos meninos e 0,97 nas meninas para a massa livre de gorduras. Em outro estudo Okasora et a ${ }^{(27)}$ avaliaram 104 crianças pela bioimpedância elétrica e pelo DEXA para mensurar o percentual de gordura corporal, a massa livre de gordura e a massa de gordura. Os autores encontraram um coeficiente de correlação de 0,90 para o percentual de gordura corporal, 0,95 para massa livre de gordura e 0,95 para a massa de gordura. Os autores concluíram que a bioimpedância elétrica deve ser utilizada para analisar a composição corporal de crianças, mas destacam a importância de se respeitar o protocolo de mensuração para não haver erros de medida.

Em outro estudo ${ }^{(28)}$ que comparou o percentual de gordura corporal medido pela bioimpedância elétrica e pelo DEXA, os autores encontraram uma baixa correlação $(0,30)$ entre os dois métodos. Nesse estudo, os pesquisadores concluem que a bioimpedância elétrica é limitada para estimar a composição corporal e que o IMC e as pregas cutâneas devem ser usados de modo preferencial.

\section{Antropometria}

A antropometria consiste na avaliação das dimensões físicas e da composição global do corpo humano. Esta técnica tem sido a mais utilizada para o diagnóstico nutricional em nível populacional, principalmente na infância e na adolescência, pela facilidade de execução e inocuidade ${ }^{(29)}$.

\section{Índice de massa corporal}

O IMC resulta da divisão do peso corporal, em quilos, pela altura em metros ao quadrado. No âmbito epidemiológico, os valores de IMC são os mais utilizados para a análise da composição corporal, mas sua interpretação no contexto individual deve ser feita com cautela. Nessa perspectiva, é importante salientar que os valores de IMC não são mais do que uma manipulação matemática das medidas de peso corporal e de estatura baseada no pressuposto de que toda medida de peso corporal que excede os indicadores de referência deverá oferecer indicações do excesso de gordura corporal. Ou seja, um maior acúmulo de gordura corporal frequentemente induz a um aumento nas medidas do peso corporal e, por sua vez, nos valores do IMC ${ }^{(24)}$.

Um estudo realizado nos $\mathrm{EUA}^{(30)}$, com 373 crianças com idade entre cinco e nove anos de idade, comparou o percentual de gordura corporal medida pelo DEXA com o IMC. Os autores observaram correlação de 0,94 em meninos e 0,92 nas meninas. Eisenman $e t a^{(28)}$ avaliaram 75 crianças com idade 
entre três e oito anos e encontraram correlação de 0,75 entre o porcentual de gordura corporal medido pelo IMC e pelo DEXA, correlação de 0,61 entre o IMC e a massa livre de gordura e de 0,85 para a massa de gordura e o IMC. Pecoraro et $a^{(31)}$ tiveram como objetivo comparar a massa de gordura medida pela bioimpedância elétrica e sua correlação com o IMC. Os autores avaliaram 228 crianças de seis anos de idade e encontraram $\mathrm{r}=0,92$ entre os dois métodos.

O IMC é um instrumento muito importante para avaliar a composição corporal em crianças, principalmente em estudos epidemiológicos com grande amostragem, por se tratar de um método barato, fácil de mensurar e não invasivo. Entretanto, para crianças hospitalizadas, seu uso como indicador de adiposidade é limitado, pois um baixo valor de IMC não indicará perda de gordura corporal, mas de massa magra. Portanto, para avaliar a composição corporal de crianças hospitalizadas, devem-se utilizar outros métodos.

\section{Pregas cutâneas}

A maior proporção de gordura corporal é localizada no tecido subcutâneo e, dessa forma, a mensuração da sua espessura é utilizada como indicador de quantidade de gordura corporal localizada em determinada região do corpo. Como a disposição da gordura localizada no tecido subcutâneo não é uniforme por toda a superfície corporal, as medidas de espessura das pregas cutâneas devem ser realizadas em várias regiões a fim de se obter uma visão mais clara sobre sua disposição ${ }^{(24)}$.

A medida de pregas cutâneas tem sido muito utilizada para estimar a gordura corporal em situações de campo e clínicas devido à sua fácil utilização, elevada precisão e custo relativamente baixo em comparação às outras técnicas ${ }^{(3)}$. Sua exatidão e precisão dependem do tipo de compasso utilizado, da familiarização dos avaliadores com as técnicas de medida e da perfeita identificação do ponto anatômico a ser medido ${ }^{(24)}$.

Daniels et al ${ }^{(32)}$ avaliaram 201 crianças e adolescentes com idade entre sete e 17 anos, comparando a distribuição da gordura corporal pelo DEXA e pelas pregas cutâneas tricipital, subescapular e suprailíaca. Encontrou-se uma correlação de 0,68, 0,80 e 0,77, respectivamente. Em ambos os sexos, a prega cutânea subescapular foi a que obteve maior correlação com a distribuição de gordura corporal medida pelo DEXA.

Um estudo italiano ${ }^{(31)}$ realizado com 228 crianças de seis anos de idade comparou a massa de gordura mensurada pela bioimpedância elétrica e pela prega cutânea tricipital, encontrando $r=0,79$. Nesse estudo, os autores não relatam a realização de protocolo específico para medir a gordura pela bioimpedância elétrica e não informam a equação utilizada para mensurar a gordura corporal por meio da prega cutânea tricipital. Portanto, deve-se avaliar com cautela o resultado encontrado.

Comparando o percentual de gordura corporal medido pelo DEXA ao medido pelas pregas cutâneas tricipital e subescapular em 75 crianças, Eisenmann et al (28) observaram correlação de 0,82 entre os métodos. Ressalta-se que a equação de Deurenberg et al ${ }^{(33)}$ foi utilizada para o cálculo do porcentual de gordura corporal por meio dos valores das pregas cutâneas tricipital e subescapular.

As equações mais utilizadas para cálculo do porcentual de gordura corporal por meio das pregas cutâneas são as propostas por Deurenberg et al ${ }^{(33)}$, que consideram sexo, etnia, idade e estágio de maturação sexual (pré-púbere ou pós-púbere), e a equação proposta por Slaughter $e t$ al ${ }^{(34)}$, na qual são considerados gênero e etnia.

\section{Medidas de perímetros}

As medidas de perímetro mais utilizadas na avaliação da composição corporal de crianças são: circunferência da cintura e relação cintura/quadril. A preocupação com o padrão de distribuição regional da gordura corporal justifica-se em razão da associação entre complicações para a saúde decorrentes de disfunções metabólicas e cardiovasculares e um maior acúmulo de gordura na região central do corpo, independentemente da idade e da quantidade total de gordura corporal ${ }^{(35)}$.

Vários estudos sobre diagnóstico de obesidade e determinação de tipo de distribuição da gordura utilizam, de forma simultânea ou não, o IMC, a circunferência da cintura e a relação cintura/ quadril ${ }^{(36,37)}$. Estudos mostram que a circunferência da cintura pode ser um instrumento mais seguro para determinar a adiposidade central tanto em adultos como em crianças ${ }^{(38)}$.

Daniels $e t$ al ${ }^{(32)}$ avaliaram a distribuição da gordura corporal pelo DEXA, a circunferência da cintura e a relação cintura/quadril. Os autores encontraram um coeficiente de correlação de 0,80 para a circunferência da cintura e 0,39 para a relação cintura/quadril, sendo que a correlação das duas medidas foi maior nas meninas.

Taylor $e t a^{(38)}$ analisaram a sensibilidade da circunferência da cintura e da relação cintura/quadril em comparação à gordura abdominal medida pelo DEXA em 580 crianças e adolescentes entre três e 19 anos de idade. A área abaixo da curva ROC (Receiver Operating Characteristics Curve) para a circunferência da cintura em meninas e meninos foi significativamente maior do que a área para a relação cintura/quadril (área abaixo da curva de 0,97 para a circunferência da cintura em ambos os sexos e área baixo da curva de 0,73 e de 0,71 para a relação cintura/quadril em meninas e meninos). 
Em estudo realizado no Brasil, Soar $e^{\text {a }}$ (l $^{(37)}$ avaliaram 419 crianças entre sete e nove anos de idade e correlacionaram o IMC com a circunferência da cintura e a relação cintura/ quadril dos escolares, encontrando correlação de 0,87 entre IMC e circunferência da cintura e de 0,46 entre IMC e relação cintura/quadril.

Uma grande limitação para a utilização da circunferência da cintura em crianças é a inexistência de um ponto de corte recomendado mundialmente para avaliar o risco de desenvolvimento de doenças cardiovasculares e metabólicas.

\section{Índice de conicidade}

O índice de conicidade baseia-se no pressuposto de que o perfil morfológico do corpo humano, ao apresentar maior concentração de gordura corporal na região central, mostra um formato de duplo cone com uma base comum, ao passo que, ao evidenciar menores quantidades de gordura na região central do corpo, tem aparência similar a um cilindro ${ }^{(24,39)}$. Para o seu cálculo, são envolvidas as medidas de circunferência da cintura e estatura em metros (m) e peso corporal em quilos (kg).

Encontrou-se apenas um estudo que avaliou a correlação do índice de conicidade com a gordura abdominal medida pelo $\mathrm{DEXA}^{(38)}$, no qual se notou uma área abaixo da curva ROC de 0,80 para as meninas e de 0,81 para os meninos. O estudo concluiu que a circunferência da cintura deve ser utilizada para mensurar a gordura corporal abdominal devido à sua facilidade, rapidez e praticidade. Para adultos, o ponto de corte de 1,73 indica risco de desenvolvimento de doenças cardiovasculares e metabólicas ${ }^{(40)}$, porém, não existe um valor recomendado para crianças, o que limita a sua utilização nesta faixa etária.

\section{Relação cintura/estatura}

A relação cintura/estatura é calculada dividindo-se a circunferência da cintura $(\mathrm{cm})$ pela medida da estatura

\section{Referências bibliográficas}

1. Lohman TG. Advances in body composition assessment. Champaign (IL): Human Kinetics; 1992.

2. Paiva CR, Gaya AC, Bottaro M, Netor JT. Bioimpedância vs absortometria radiológica de dupla energia na avaliação da composição corporal em crianças. Unimontes Científica 2002;3:23-8.

3. Heyward VH, Stolarczyk LM. Avaliação da composição corporal. São Paulo: Manole; 2000.

4. Clarys JP, Martin AD, Drinkwater DT. Gross tissue weights in the human body by cadaver dissection. Human Biol 1984;56:459-73.

5. Martin AD, Drinkwater DT. Variability in the measures of body fat. Assumptions or technique? Sports Med 1991;11:277-88.

6. Wagner DR, Heyward VH. Techniques of body composition assessment: a review of laboratory and field methods. Res Q Exerc Sport 1999;70:135-49. (cm). Alguns autores têm demonstrado que esta relação está fortemente associada a diversos fatores de risco cardiovasculares em adultos e crianças ${ }^{(41-44)}$. A relação cintura/estatura é um indicador simples e efetivo para mensurar a obesidade abdominal tanto em adultos quanto em crianças, discriminando risco coronariano melhor do que o IMC e a circunferência da cintura, nessas faixas etárias $^{(45-47)}$. Essas pesquisas apresentam algumas razões para tal fato: a relação cintura/estatura mostrou alta correlação com a gordura visceral e com fatores de risco para doenças cardiovasculares em crianças e adultos; a relação cintura/estatura apresenta baixa correlação com idade e sexo e, sendo assim, o ponto de corte seria o mesmo para adultos e crianças. Um valor maior de 0,50 é sugerido como ponto de corte para o risco de desenvolvimento de doenças cardiovasculares em indivíduos de ambos os sexos a partir dos seis anos de idade. Tal ponto de corte não pode ser utilizado para crianças menores de cinco anos, pois estudos mostram que este valor superestima o risco de desenvolvimento de doenças cardíacas ${ }^{(46-48)}$.

\section{Considerações finais}

Por meio da revisão realizada, observa-se que existem vários métodos para se avaliar a composição corporal em crianças, cada um com suas vantagens e desvantagens. Ao definir o melhor método, deve-se eleger aquele que melhor detecte o problema que se pretende corrigir, levando em consideração os custos, o nível de treinamento dos avaliadores, o tempo de execução, a receptividade da população e os possíveis riscos à saúde que o método pode acarretar. É imprescindível, ainda, que o método escolhido seja validado para a população que se quer estudar, principalmente aqueles relacionados à antropometria.

7. Lukaski HC. Methods for assessment of human body composition: traditional and new. Am J Clin Nutr 1987;46:537-56.

8. Cintra IP, Costa RF, Fisberg M. Composição corporal na infância e adolescência. In: Fisberg M, editor. Atualização em obesidade na infância e adolescência. São Paulo: Atheneu; 2004.

9. Mcardle WD, Katch FI, Katch VL. Fisiologia do exercício, energia, nutrição e desempenho humano. $4^{\text {th }}$ ed. Rio de Janeiro: Guanabara Koogan; 1998.

10. Siri WE. Body composition from fluid spaces and density: analysis of methods. In: Brozek J, Henschel A, editors. Techniques for measuring body composition. Washington DC: National Academy of Science; 1961. p. 223-44.

11. Brozek J, Grande F, Anderson JT, Keys A. Densitometric analysis of body composition: revision of some quantitative assumptions. Ann NY Acad Sci 1963;110:113-40. 
12. Howley ET, Franks BD. Manual do instrutor de condicionamento físico para saúde. $3^{\text {rd }}$ ed. Porto Alegre: Artmed; 2000.

13. McCrory MA, Gomez TD, Bernauer EM, Molé PA. Evaluation of a new air displacement plethysmograph for measuring human body composition. Med Sci Sports Exerc 1995;27:1686-91.

14. Mello MT, Dâmaso AR, Antunes HK, Siqueira KO, Castro ML, Bertolino SV et al. Avaliação da composição corporal em adolescentes obesos: o uso de dois diferentes métodos. Rev Bras Med Esporte 2005;11:267-70.

15. Guedes DP, Guedes JE. Controle do peso corporal: composição corporal, atividade física e nutrição. Londrina: MidioGraf; 1998.

16. Fields DA, Goran MI. Body composition techniques and the four-compartment model in children. J Appl Physiol 2000;89:613-20.

17. Fields DA, Hunter GR, Goran MI. Validation of the BOD POD with hydrostatic weighing: influence of body clothing. Int $\mathrm{J}$ Obes Relat Metab Disord 2000;24:200-5.

18. Vescovi JD, Zimmerman SL, Miller WC, Hildebrandt L, Hammer RL, Fernhall B. Evaluation of the BOD POD for estimating percentage body fat in a heterogeneous group of adult humans. Eur J Appl Physiol 2001;85: 326-32.

19. Demerath EW, Guo SS, Chumlea WC, Towne B, Roche AF, Siervogel RM. Comparison of percent body fat estimates using air displacement plethysmography and hydrodensitometry in adults and children. Int $\mathrm{J}$ Obes Relat Metab Disord 2002;26:389-97.

20. Ma G, Yao M, Liu Y, Lin A, Zou H, Urlando A et al. Validation of a new pediatric air displacemet plethysmography for assessing body composition in infants. Am J Clin Nutr 2004;79:653-60.

21. Paiva CR, Gaya AC, Bottaro M, Bezerra RF. Assessment of the body composition of brazilian boys: the bioimpedence method. Rev Bras Cineantropom Desempenho Hum 2002;4:37-45.

22. Marques MB, Heyward V, Paiva CE. Validação cruzada de equações de bioimpedância em mulheres brasileiras por meio de absortometria radiológica de dupla energia (DXA). Rev Bras Cien Movim 2000;8:14-20.

23. Sopher AB, Thornton JC, Wang J, Pierson RN, Heymsfield SB, Horlick M. Measurement of percentage of body fat in 411 children and adolescents: a comparison of dual-energy $\mathrm{X}$-ray absorptiometry with a four compartment model. Pediatrics 2004;113:1285-90.

24. Guedes DP. Recursos antropométricos para análise da composição corporal. Rev Bras Educ Fís Esp 2006;20:115-9.

25. Kushner RF. Bioeletrical impendance analysis: a review of principles and applications. J Am Coll Nutr 1992;11:199-209.

26. Wu YT, Nielsen DH, Cassady SL, Cook JS, Janz KF, Hansen JR. Crossvalidation of biolectrical impedance analysis of body composition in children and adolescents. Phys Ther 1993;73:320-7.

27. Okasora K, Takaya R, Tokuda M, Fukunaga $\mathrm{Y}$, Oguni T, Tanaka $\mathrm{H}$ et al. Comparison of bioelectrical impedance analysis and dual energy $\mathrm{X}$-ray absorptiometry for assessment of body composition in children. Pediatr Int 1999;41:121-5.

28. Eisenmann JC, Heelan KA, Welk GJ. Assessing body composition among 3-to-8-year-old children: anthropometry, BIA, and DXA. Obes Res 2004;12: 1633-40.

29. Sigulem DM, Devincenzi UM, Lessa AC. Diagnosis of the nutritional status of children and adolescents. J Pediatr (Rio J) 2000;76 (Suppl 3):S275-84.

30. Lindsay RS, Hanson RL, Roumain J, Ravussin E, Knowler WC, Tataranni PA. Body mass index as a measure of adiposity in children and adolescents: relationship to adiposity by dual energy $\mathrm{x}$-ray absorptiometry and to cardiovascular risk factors. J Clin Endocrinol Metab 2001;86:4061-7.
31. Pecoraro P, Guida B, Caroli M, Trio R, Falconi C, Principato S et al. Body mass index and skinfold thickness versus bioimpedance analysis: fat mass prediction in children. Acta Diabetol 2003;40:S278-81.

32. Daniels SR, Khoury PR, Morrison JA. Utility of different measures of body fat distribution in children and adolescents. Am J Epidemiol 2000;152: 1179-84.

33. Deurenberg P, Pieters JJ, Hautvast JG. The assessment of the body fat percentage by skinfold thickness measurements in childhood and young adolescence. Br J Nutr 1990;63:293-303.

34. Slaughter MH, Lohman TG, Boileau RA, Horswill CA, Stillman RJ, Van Loan $\mathrm{MD}$ et al. Skinfold equations for estimations of body fatness in children and youth. Hum Biol 1988;60:709-23.

35. Thomas GN, Ho SY, Lam KS, Janus ED, Hedley AJ, Lam TH et al. Impact of obesity and body fat distribution on cardiovascular risk factors in Hong Kong Chinese. Obes Res 2004;12:1805-13.

36. Maffeis C, Grezzani A, Pietrobelli A, Provera S, Tatò L. Does waist circumference predict fat gain in children? Int J Obes Relat Metab Disord 2001;25:978-83.

37. Soar C, Vasconcelos FA, Assis MA. Waist-hip ratio and waist circumference associated with body mass index in a study with schoolchildren. Cad Saude Publica 2004;20:1609-16.

38. Taylor RW, Jones IE, Williams SM, Goulding A. Evaluation of waist circumference, waist to hip ratio and the conicity index as screening tools for high trunk fat mass, as measured by dual energy $\mathrm{x}$-ray absorptiometry, in children aged 3-19y. Am J Clin Nutr 2000;72:490-5.

39. Valdez R. A simple model-based index of abdominal adiposity. J Clin Epidemiol 1991;44:955-6.

40. Valdez R, Seidell JC, Ahn YI, Weiss KM. A new index of abdominal adiposity as an indicator of risk for cardiovascular disease. A cross-population study. Int J Obes Relat Metab Disord 1993;17:77-82.

41. Lin WY, Lee LT, Chen CY, Lo H, Hsia HH, Liu IL et al. Optimal cut-off values for obesity: using simple anthropometric indices to predict cardiovascular risk factors in Taiwan. Int J Obes Relat Metab Disord 2002;26:1232-8.

42. Ho SY, Lam TH, Janus ED. Waist to stature ratio is more strongly associated with cardiovascular risk factors than other simple anthropometric indices. Ann Epidemiol 2003;13:683-91.

43. Pitanga FJ, Lessa I. Razão cintura-estatura como discriminador do risco coronariano de adultos. Rev Assoc Med Bras 2006;52:157-61.

44. Freedman DS, Kahn HS, Mei Z, Grummer-Strawn LM, Dietz WH, Srinivasan SR et al. Relation of body mass index and waist- to-height ratio to cardiovascular disease risk factors in children and adolescents: the Bogalusa Heart Study. Am J Clin Nutr 2007;86:33-40.

45. Zhu S, Wang Z, Heshka S, Heo M, Faith MS, Heymsfield SB. Waist circumference and obesity-associated risk factors among whites in the third National Health and Nutrition Examination Survey: clinical action thresholds. Am J Clin Nutr 2002;76:743-9.

46. Savva SC, Tornaritis M, Savva ME, Kourides Y, Panagi A, Silikiotou N et al. Waist circumference and waist-to-height ratio are better predictors of cardiovascular disease risk factors in children than body mass index. Int $\mathrm{J}$ Obes Relat Metab Disord 2000;24:1453-8.

47. Li C, Ford ES, Mokdad AH, Cook S. Recent trends in waist circumference and waist-height ratio among US children and adolescents. Pediatrics 2006;118:e1390-8.

48. Hsieh SD, Yoshinaga H, Muto T. Waist-to-height ratio, a simple and practical index for assessing central fat distribution and metabolic risk in Japanese men and women. Int J Obes Relat Metab Disord 2003;27:610-6. 